\title{
THE M/M/c QUEUE WITH MASS EXODUS AND MASS ARRIVALS WHEN EMPTY
}

\author{
LINA ZHANG ${ }^{* * *}$ AND \\ JUNPING LI, ${ }^{* * *}$ Central South University
}

\begin{abstract}
In this paper we consider an $\mathrm{M} / \mathrm{M} / c$ queue modified to allow both mass arrivals when the system is empty and the workload to be removed. Properties of queues which terminate when the server becomes idle are firstly developed. Recurrence properties, equilibrium distribution, and equilibrium queue-size structure are studied for the case of resurrection and no mass exodus. All of these results are then generalized to allow for the removal of the entire workload. In particular, we obtain the Laplace transformation of the transition probability for the absorptive $\mathrm{M} / \mathrm{M} / c$ queue.
\end{abstract}

Keywords: $\mathrm{M} / \mathrm{M} / c$ queue; equilibrium distribution; idle time; queue size; recurrence

2010 Mathematics Subject Classification: Primary 60J35

Secondary $60 \mathrm{~J} 27 ; 60 \mathrm{~K} 25$

\section{Introduction}

Markovian queue theory is a basic and important branch of queueing theory. It interweaves the general theory of queueing models and the general theory and applications of continuoustime Markov chains and has become a very successful and fruitful research field. There are numerous works in the literature; see, for example, Asmussen [3] and Gross and Harris [15] for queueing models, and Anderson [1] for applications of continuous-time Markov chains. See also Chen [9], [10], which contain much new material concerning continuous-time Markov chains.

Markovian queueing models with state-independent and state-dependent controls have also attracted considerable research interest. For a recent work in this direction, see Chen $e t$ al. [8]. In these models, arbitrary inputs are allowed when the queue is empty. Usually, this is due to the consideration of improving working efficiency. Gelenbe [13] and Gelenbe et al. [14] introduced the particularly interesting concept of negative arrivals; see also Bayer and Boxma [4] and Jain and Sigman [16]. Parthasarathy and Krishna Kumar [18] allowed arbitrary input when the queue is empty, and Chen and Renshaw [6], [7] introduced the possibility of removing the entire workload. Di Crescenzo et al. [11] considered the first effective catastrophe occurrence time of a birth-death process. Dudin and Karolik [12] investigated a BMAP/SM/1 system which is exposed to disaster arrivals. The $\mathrm{M}_{t} / \mathrm{M} / t / \mathrm{N}$ queue with catastrophes can be seen in Zeifman and Korotysheva [19]. From a practical point of view, models with disasters are quite interesting, for example, the work of hardware influenced by breaks and occasional power disappearance, the work of communication systems influenced by computer viruses or

Received 22 July 2014; revision received 2 November 2014.

* Postal address: School of Mathematics and Statistics, Central South University, Changsha, 410075,

Hunan Province, P. R. China.

** Email address: zln514@163.com

*** Email address: jpli@mail.csu.edu.cn 
intentional external interventions, deletion of transactions in databases, the operation of air defense radars, etc. More detailed information about the real and potential applications and corresponding descriptions can be found in [2].

It is worth noting that $\mathrm{Di}$ Crescenzo et al. [11] considered the Laplace transform of the probability density function of the catastrophe's first occurrence time, while in this paper we investigate the extinction, recurrence, and ergodicity properties of a modified $M / M / c$ queue. Also note that in [6] and [8] the queues with a single server in the system were discussed. In this paper we consider $c$-servers in the system, it is a natural generalization of the models considered in [6] and [8]. Since there is more than one server in the system, the method in [6] and [8] fails and we have to find alternative techniques and methods to treat it. Moreover, in order to consider the recurrence properties, equilibrium distribution, and equilibrium queue-size structure of the modified $\mathrm{M} / \mathrm{M} / c$ queue, we have to first show all the Laplace transformations of the transition probability for the absorptive $\mathrm{M} / \mathrm{M} / c$ queue. However, it is very difficult to obtain the transition probability or transition function for a general Markov process.

The infinitesimal behavior of our model is described by a $q$-matrix $Q=\left\{q_{i j} ; i, j \geq 0\right\}$ which splits into

$$
Q=Q^{*}+Q_{s}+Q_{d}
$$

where $Q^{*}=\left\{q_{i j}^{*} ; i, j \geq 0\right\}, Q_{s}=\left\{q_{i j}^{(s)} ; i, j \geq 0\right\}$, and $Q_{d}=\left\{q_{i j}^{(d)} ; i, j \geq 0\right\}$ are all conservative $q$-matrices which are given as follows

$$
\begin{aligned}
& q_{i j}^{*}= \begin{cases}\min (i, c) a & \text { if } i \geq 1, j=i-1, \\
-b-\min (i, c) a & \text { if } i \geq 1, j=i, \\
b & \text { if } i \geq 1, j=i+1, \\
0 & \text { otherwise, }\end{cases} \\
& q_{i j}^{(s)}= \begin{cases}-h & \text { if } i=0, j=0, \\
h_{j} & \text { if } i=0, j \geq 1, \\
0 & \text { otherwise, }\end{cases} \\
& q_{i j}^{(d)}= \begin{cases}\beta & \text { if } i \geq 1, j=0, \\
-\beta & \text { if } i \geq 1, j=i, \\
0 & \text { otherwise, }\end{cases}
\end{aligned}
$$

respectively. Here $a \geq 0, b>0, \beta \geq 0$, and $h_{j} \geq 0, j \geq 1$ with $0 \leq h:=\sum_{j=1}^{\infty} h_{j}<\infty$. Furthermore, denote $\tilde{Q}=Q^{*}+Q_{s}$.

Definition 1.1. Let $Q=\left(q_{i j} ; i, j \in \mathbb{Z}_{+}\right)$be defined in (1.1)-(1.4). The corresponding transition function $\mathbb{P}(t)=\left(p_{i j}(t) ; i, j \in \mathbb{Z}_{+}\right)$is called the modified $\mathrm{M} / \mathrm{M} / c$ queueing process.

This process includes several interesting models as special cases. For example, if $c=1$, we recover the model considered in Chen and Renshaw [6]. Whilst if $c=1$ and $\beta=0$ (i.e. no annihilation), $h_{1}=b$ and $h_{j}=0, j \geq 2$, we obtain the ordinary $\mathrm{M} / \mathrm{M} / 1$ queue.

The structure of this paper is organized as follows. We first introduce some key lemmas and consider the properties of the stopped $\mathrm{M} / \mathrm{M} / c$ queue in Section 2 , since our later discussion depends heavily upon them. The $\mathrm{M} / \mathrm{M} / c$ queue, modified to ensure that arbitrary mass arrivals may occur when the system is empty, is fully discussed in Section 3. Whilst the effect of removing the entire workload is studied in Section 4. 


\section{The stopped $M / M / c$ queue}

We first consider the $\mathrm{M} / \mathrm{M} / c$ queue, modified by terminating the process when it first becomes empty. The associated $q$-matrix $Q^{*}$ is given by (1.2). Define the generating functions

$$
B(s)=a-(a+b) s+b s^{2}, \quad B_{i}(s)=B(s)+(i-1) a(1-s), \quad i=1,2, \ldots, c,
$$

and denote

$$
U(s):=B_{c}(s)=b s^{2}-(b+c a) s+c a .
$$

These are all well defined for $s \in[-1,1]$, whilst as a power series they are $C^{\infty}$ functions with respect to $s$ on $(-1,1)$. Also, $B(\cdot)$ and $U(\cdot)$ are convex functions on $[0,1]$. The convex property of $U(\cdot)$ immediately yields the following simple, yet important, result.

Lemma 2.1. The equation $U(s)=0$ has the smallest root $u=\left(b+c a-\sqrt{(b-c a)^{2}}\right) / 2 b$ on $[0,1]$ with $u=1$ if $b \leq c a$ and $u=c a / b<1$ if $b>c a$.

We now define, for any $\lambda>0$,

$$
U_{\lambda}(s):=U(s)-\lambda s=b s^{2}-(\lambda+b+c a) s+c a .
$$

It is clear that $U_{\lambda}(\cdot)$ is also $C^{\infty}$ with respect to $s$ on $(-1,1)$ and that it is convex on $[0,1]$. The following result can be easily proved.

Lemma 2.2. For any fixed $\lambda>0$, the equation $U_{\lambda}(s)=0$ has exactly one root

$$
u(\lambda)=\frac{b+c a+\lambda-\sqrt{(b+c a+\lambda)^{2}-4 b c a}}{2 b}
$$

on $[0,1]$, and $0<u(\lambda)<1$.

Similar to the proof of [7, Lemma 2.3], we obtain the following lemma.

Lemma 2.3. For $u(\cdot)$ as defined in Lemma 2.2. (i) $u(\lambda) \in C^{\infty}(0, \infty)$;

(ii) $u(\lambda)$ is a decreasing function of $\lambda>0$;

(iii) $u(\lambda) \downarrow 0$ and $\lambda u(\lambda) \rightarrow$ ca as $\lambda \rightarrow \infty$;

(iv) when $\lambda \rightarrow 0^{+}$,

$$
u(\lambda) \uparrow u= \begin{cases}1 & \text { if } b \leq c a, \\ \frac{c a}{b}<1 & \text { if } b>c a,\end{cases}
$$

where $u$ is the smallest root of $U(s)=0$ on $[0,1]$;

(v) for any positive integer $k$,

$$
\lim _{\lambda \rightarrow 0^{+}} \frac{1-u(\lambda)^{k}}{\lambda}= \begin{cases}\infty & \text { if } b \geq c a, \\ \frac{k}{c a-b} & \text { if } b<c a .\end{cases}
$$

Let $\left(p_{i j}^{*}(t) ; i, j \geq 0\right)$ and $\left(\phi_{i j}^{*}(\lambda) ; i, j \geq 0\right)$ be the $Q^{*}$-function and $Q^{*}$-resolvent, respectively. 
Theorem 2.1. For any $i \geq 0, \phi_{i j}^{*}(\lambda)(0 \leq j \leq c-1)$ is the unique solution of the following linear equations:

$$
\begin{gathered}
-\lambda \phi_{i 0}^{*}(\lambda)-\sum_{k=1}^{c-1} u(\lambda)^{k-1}\left[B_{c}(u(\lambda))-B_{k}(u(\lambda))\right] \phi_{i k}^{*}(\lambda)=-u(\lambda)^{i} \\
-\lambda \phi_{i 0}^{*}(\lambda)+a \phi_{i 1}^{*}(\lambda)=-\delta_{i 0} \\
(-b-a-\lambda) \phi_{i 1}^{*}(\lambda)+2 a \phi_{i 2}^{*}(\lambda)=-\delta_{i 1}, \\
\vdots \\
\phi_{i j-1}^{*}(\lambda) b+(-b-j a-\lambda) \phi_{i j}^{*}(\lambda)+(j+1) a \phi_{i j+1}^{*}(\lambda)=-\delta_{i j} \\
\vdots \\
\phi_{i c-3}^{*}(\lambda) b+[-b-(c-2) a-\lambda] \phi_{i c-2}^{*}(\lambda)+(c-1) a \phi_{i c-1}^{*}(\lambda)=-\delta_{i c-2},
\end{gathered}
$$

where $u(\lambda)(\lambda>0)$ is the unique root of $U_{\lambda}(s)=0$ on $[0,1]$.

Proof. By the Kolmogorov forward equations, we have

$$
\begin{gathered}
-\lambda \phi_{i 0}^{*}(\lambda)+a \phi_{i 1}^{*}(\lambda)=-\delta_{i 0}, \\
(-b-a-\lambda) \phi_{i 1}^{*}(\lambda)+2 a \phi_{i 2}^{*}(\lambda)=-\delta_{i 1}, \\
\vdots \\
\phi_{i c-1}^{*}(\lambda) b+(-b-c a-\lambda) \phi_{i c}^{*}(\lambda)+c a \phi_{i c+1}^{*}(\lambda)=-\delta_{i c}, \\
\phi_{i j-1}^{*}(\lambda) b+(-b-c a-\lambda) \phi_{i j}^{*}(\lambda)+c a \phi_{i j+1}^{*}(\lambda)=-\delta_{i j}, \quad j \geq c+1 .
\end{gathered}
$$

Thus,

$$
\lambda \sum_{j=0}^{\infty} \phi_{i j}^{*}(\lambda) s^{j}-s^{i}=\sum_{k=1}^{c-1} s^{k-1} B_{k}(s) \phi_{i k}^{*}(\lambda)+B_{c}(s) \sum_{j=c}^{\infty} \phi_{i j}^{*}(\lambda) s^{j-1}
$$

Since $\lambda u(\lambda)=B_{c}(u(\lambda))$, we then have

$$
-\lambda \phi_{i 0}^{*}(\lambda)-\sum_{k=1}^{c-1} u(\lambda)^{k-1}\left[B_{c}(u(\lambda))-B_{k}(u(\lambda))\right] \phi_{i k}^{*}(\lambda)=-u(\lambda)^{i}
$$

Combining (2.8) with the first $c-1$ equations of (2.7), we obtain the unique $\phi_{i j}^{*}(\lambda), i \geq 0,0 \leq$ $j \leq c-1$. Thus, the proof is complete.

Remark 2.1. As usual, it is very difficult to obtain the transition probability or transition function $p_{i j}^{*}(t)$ for a general Markov process. We find it particularly noteworthy that we can obtain the resolvent $\phi_{i j}^{*}(\lambda), i \geq 0,0 \leq j \leq c-1$ of the transition probability $p_{i j}^{*}(t)$ from Theorem 2.1. Furthermore, by carefully checking the proof of Theorem 2.1 and using (2.7), we obtain all the resolvent $\phi_{i j}^{*}(\lambda), i \geq 0$ of the transition probability $p_{i j}^{*}(t)$. 
By the Kolmogorov forward equation $\Phi^{*}(\lambda)\left(\lambda I-Q^{*}\right)=I$ and Lemma 2.2 , we obtain the following theorem.

Theorem 2.2. The generating functions of the $Q^{*}$-resolvent take the form

$$
\begin{aligned}
L_{i}(\lambda, s) & :=\sum_{j=0}^{\infty} \phi_{i j}^{*}(\lambda) s^{j} \\
& =\frac{U(s) \phi_{i 0}^{*}(\lambda)+\sum_{k=1}^{c-1} \phi_{i k}^{*}(\lambda) s^{k}\left[U(s)-B_{k}(s)\right]-s^{i+1}}{U_{\lambda}(s)}, \quad i \geq 0,
\end{aligned}
$$

where $U(s)$ and $U_{\lambda}(s)$ are defined in (2.2) and (2.3), respectively, and $\phi_{i k}^{*}(\lambda), i \geq 0,1 \leq k \leq$ $c-1$, can be obtained by Theorem 2.1. In particular, $L_{0}(\lambda, s)=1 / \lambda$, that is, $\phi_{00}^{*}(\lambda)=1 / \lambda$, whilst, for $i, j \geq 1$,

$$
\phi_{i 0}^{*}(\lambda)=\frac{u(\lambda)^{i}-\sum_{k=1}^{c-1} \phi_{i k}^{*}(\lambda) u(\lambda)^{k-1}(c-k) a(1-u(\lambda))}{\lambda}, \quad \phi_{0 j}^{*}(\lambda)=0,
$$

where $u(\lambda), \lambda>0$ is the unique root of $U_{\lambda}(s)=0$ on $[0,1]$ and thereby possesses the properties of Lemma 2.3.

Similar to that considered in [17, Lemma 3.1], we immediately obtain the following lemma.

Lemma 2.4. For any $i \geq 0$, we have

$$
\int_{0}^{\infty} p_{i k}^{*}(t) \mathrm{d} t<\infty, \quad k \geq 1
$$

Let $\left\{X_{t} ; t \geq 0\right\}$ denote the $Q^{*}$-process. Define the extinction time as

$$
\tau_{0}:= \begin{cases}\inf \left\{t>0 ; X_{t}=0\right\} & \text { if } X_{t}=0 \text { for some } t>0 \\ \infty & \text { if } X_{t}>0 \text { for all } t>0\end{cases}
$$

and $w_{k}(t):=\mathbb{P}_{r}\left(\tau_{0} \leq t \mid X_{0}=k\right)$. From (2.10), we immediately obtain the following conclusion.

Lemma 2.5. Suppose that the $Q^{*}$-process $\left\{X_{t} ; t \geq 0\right\}$ starts from $X_{0}=k>0$. Then the Laplace transform of $w_{k}(t)$ is

$$
\int_{0}^{\infty} \mathrm{e}^{-\lambda t} \mathbb{P}_{r}\left(\tau_{0} \leq t \mid X_{0}=k\right) \mathrm{d} t=\frac{u(\lambda)^{k}-\sum_{i=1}^{c-1} \phi_{k i}^{*}(\lambda) u(\lambda)^{i-1}(c-i) a(1-u(\lambda))}{\lambda}
$$

where $u(\lambda)$ is the unique root of $U_{\lambda}(s)=0$ on $[0,1]$ and thereby possesses the properties in Lemma 2.3 , and $\phi_{k i}^{*}(\lambda), k \geq 1,1 \leq i \leq c-1$, can be obtained by Theorem 2.1 .

Theorem 2.3. Consider the $Q^{*}$-process $\left\{X_{t} ; t \geq 0\right\}$. Denote the extinction probability $e_{k}=$ $\mathbb{P}_{r}\left(\tau_{0}<\infty \mid X_{0}=k\right)=\lim _{t \rightarrow \infty} p_{k 0}^{*}(t), k \geq 1$, and $m_{i}(k)=\int_{0}^{\infty} p_{k i}^{*}(t) \mathrm{d} t, i \geq 1$.

(i) If $b \leq c a$, then for any $k \geq 1, e_{k}=1$; 
(ii) if $b>c a$, then for any $k \geq 1, e_{k}$ and $m_{i}(k), 1 \leq i \leq c-1$, is the unique solution of the following linear equations:

$$
\begin{gathered}
e_{k}=u^{k}-\sum_{i=1}^{c-1} m_{i}(k) u^{i-1}(c-i) a(1-u), \\
a m_{1}(k)=e_{k} \\
(-b-a) m_{1}(k)+2 a m_{2}(k)=-\delta_{k 1}, \\
\vdots \\
b m_{j-1}(k)+(-b-j a) m_{j}(k)+(j+1) a m_{j+1}(k)=-\delta_{k j}, \\
\vdots \\
b m_{c-3}(k)+[-b-(c-2) a] m_{c-2}(k)+(c-1) a m_{c-1}(k)=-\delta_{k c-2},
\end{gathered}
$$

where $u$ is the smallest root of $U(s)=0$ on $[0,1]$ with $u=1$ if $b \leq c a$ and $u<1$ if $b>c a$. Moreover, all the $m_{i}(k), k \geq 1, i \geq 1$, can be obtained.

(iii) The mean extinction time is

$$
\mathbb{E}\left(\tau_{0} \mid X_{0}=k\right)= \begin{cases}\frac{1}{c a-b}\left[k+\sum_{i=1}^{c-1} m_{i}(k)(c-i) a\right] & \text { if } b<c a, \\ \infty & \text { if } b \geq c a,\end{cases}
$$

where $m_{i}(k), 1 \leq i \leq c-1$, can be obtained by (2.12).

Proof. Using Lemma 2.4, Lemma 2.5, (2.4), and (2.11), in combination with the Tauberian theorem, yields

$$
\begin{aligned}
e_{k} & =\lim _{\lambda \rightarrow 0^{+}} \lambda \phi_{k 0}^{*}(\lambda) \\
& =\lim _{\lambda \rightarrow 0^{+}}\left(u(\lambda)^{k}-\sum_{i=1}^{c-1} \phi_{k i}^{*}(\lambda) u(\lambda)^{i-1}(c-i) a(1-u(\lambda))\right) \\
& = \begin{cases}1 & \text { if } b \leq c a, \\
u^{k}-\sum_{i=1}^{c-1} m_{i}(k) u^{i-1}(c-i) a(1-u)<1 & \text { if } b>c a\end{cases}
\end{aligned}
$$

thus, Theorem 2.3(i) is proven. By (2.4), (2.11), and letting $\lambda \rightarrow 0^{+}$in every equation of (2.6), we immediately obtain (2.12). Combining (2.12) with (2.7), we obtain all the $m_{i}(k), k \geq$ $1, i \geq 1$, which completes the proof of Theorem 2.3(ii).

Using the Tauberian theorem once again and in conjunction with (2.4) and (2.5), we obtain

$$
\begin{aligned}
\mathbb{E}\left(\tau_{0} \mid X_{0}=k\right) & =\int_{0}^{\infty} \mathbb{P}_{r}\left(\tau_{0}>t \mid X_{0}=k\right) \mathrm{d} t \\
& =\lim _{\lambda \rightarrow 0^{+}} \frac{1-\lambda \phi_{k 0}^{*}(\lambda)}{\lambda} \\
& = \begin{cases}\frac{1}{c a-b}\left[k+\sum_{i=1}^{c-1} m_{i}(k)(c-i) a\right] & \text { if } b<c a, \\
\infty & \text { if } b \geq c a ;\end{cases}
\end{aligned}
$$

thus, Theorem 2.3(iii) is proven. The proof is complete. 


\section{The $M / M / c$ queue with resurrection}

We now extend the process by allowing mass arrivals of size $j$ to occur at rate $h_{j}$ when the queue is empty and $\beta=0$. So the $q$-matrix is $\tilde{Q}=Q^{*}+Q_{s}$, where $Q_{s}$ is given by (1.3). In addition to the generating functions $B(s), B_{i}(s)$, and $U(s)$ defined in (2.1) and (2.2), we need to define

$$
H(s):=\sum_{j=1}^{\infty} h_{j} s^{j} .
$$

Obviously, $H(s)$ is well defined on $[-1,1]$ and $H(1)=h>0$. Moreover, denote

$$
\mu_{1}=H^{\prime}(1)=\sum_{j=1}^{\infty} j h_{j},
$$

note that $\mu_{1}$ is not currently presumed to be finite.

Let $\tilde{R}(\lambda)=\left\{\tilde{r}_{i j}(\lambda) ; i, j \geq 0\right\}$ denote the $\tilde{Q}$-resolvent. Similar to the proof of [7, Theorem 3.1], using the resolvent decomposition theorem (see [5]), we have the following conclusion.

Theorem 3.1. For $\tilde{R}(\lambda)=\left\{\tilde{r}_{i j}(\lambda) ; i, j \geq 0\right\}$, we have

$$
\begin{aligned}
& \tilde{r}_{00}(\lambda)=\left[\lambda+\lambda \sum_{i=1}^{\infty} \sum_{j=1}^{\infty} h_{i} \phi_{i j}^{*}(\lambda)\right]^{-1}, \\
& \tilde{r}_{i 0}(\lambda)=\tilde{r}_{00}(\lambda) a \phi_{i 1}^{*}(\lambda), \quad i \geq 1, \\
& \tilde{r}_{0 j}(\lambda)=\tilde{r}_{00}(\lambda) \sum_{i=1}^{\infty} h_{i} \phi_{i j}^{*}(\lambda), \quad j \geq 1, \\
& \tilde{r}_{i j}(\lambda)=\phi_{i j}^{*}(\lambda)+\tilde{r}_{i 0}(\lambda) \sum_{k=1}^{\infty} h_{k} \phi_{k j}^{*}(\lambda), \quad i, j \geq 1,
\end{aligned}
$$

where $\Phi^{*}(\lambda)=\left\{\phi_{i j}^{*}(\lambda) ; i, j \geq 0\right\}$ is the $Q^{*}$-resolvent given in Theorem 2.2.

Using Theorem 3.1 we now consider the recurrence properties of the modified $\mathrm{M} / \mathrm{M} / c$ queue determined by our current $q$-matrix $\tilde{Q}$.

Theorem 3.2. For the modified $M / M / c$ queueing process with $q$-matrix $\tilde{Q}$, we have

(i) the process is recurrent if and only if $b \leq c a$;

(ii) the process is positive recurrent if and only if $b<c a$ and $\mu_{1}<\infty$, where $\mu_{1}$ is defined by (3.1).

Proof of Theorem 3.2(i). Since the process is irreducible, it is recurrent if and only if

$$
\lim _{\lambda \rightarrow 0^{+}} \tilde{r}_{00}(\lambda)=\infty
$$

and so by (3.2) if and only if

$$
\lim _{\lambda \rightarrow 0^{+}} \sum_{i=1}^{\infty} h_{i} \sum_{j=1}^{\infty} \lambda \phi_{i j}^{*}(\lambda)=0
$$


So from (2.10), we see that (3.4) holds if and only if

$$
\lim _{\lambda \rightarrow 0^{+}}\left(u(\lambda)^{i}-\sum_{k=1}^{c-1} \phi_{i k}^{*}(\lambda) u(\lambda)^{k-1}(c-k) a(1-u(\lambda))\right)=1 \quad \text { for all } i \geq 1,
$$

which, by (2.13), is equivalent to $b \leq c a$.

Proof of Theorem 3.2(ii). Again using irreducibility and (3.2), the process is positive recurrent if and only if $\lim _{\lambda \rightarrow 0^{+}} \lambda \tilde{r}_{00}(\lambda)>0$, i.e. if and only if $\lim _{\lambda \rightarrow 0^{+}} \sum_{i=1}^{\infty} h_{i} \sum_{j=1}^{\infty} \phi_{i j}^{*}(\lambda)<\infty$. Comparison with (2.4), (2.5), (2.10), and (2.11) then shows that the process is positive recurrent if and only if $b<c a$ and $\mu_{1}=\sum_{i=1}^{\infty} i h_{i}<\infty$. The proof is complete.

Having determined conditions for our modified $\mathrm{M} / \mathrm{M} / c$ queue to be positive recurrent, we are now in a position to determine the equilibrium distribution through the generating function $\Pi(s):=\sum_{j=0}^{\infty} \pi_{j} s^{j}$.

Theorem 3.3. Under the positive recurrence conditions given in Theorem 3.2 , that is, $b<c a$ and $\mu_{1}<\infty$, the equilibrium generating function $\Pi(s)$ takes the form

$$
\Pi(s)=\pi_{0}\left[1+\frac{s(h-H(s))}{U(s)}\right]+\frac{1}{U(s)} \sum_{k=1}^{c-1} \pi_{k} s^{k}(c-k) a(1-s),
$$

where

$$
\pi_{0}=(c a-b)\left[c a-b+\mu_{1}+\sum_{k=1}^{c-1} r_{k}(c-k) a\right]^{-1}, \quad \pi_{k}=\pi_{0} r_{k}, \quad k \geq 1
$$

and $r_{k}:=\sum_{i=1}^{\infty} h_{i} m_{k}(i), k \geq 1$, satisfies

$$
\begin{gathered}
a r_{1}=h, \\
(-b-a) r_{1}+2 a r_{2}=-h_{1}, \\
\vdots \\
b r_{c-1}+(-b-c a) r_{c}+c a r_{c+1}=-h_{c}, \\
b r_{j-1}+(-b-c a) r_{j}+c a r_{j+1}=-h_{j}, \quad j \geq c+1 .
\end{gathered}
$$

Proof. Noting that $\pi_{j}=\lim _{\lambda \rightarrow 0^{+}} \lambda \tilde{r}_{0 j}(\lambda)$ for all $j \geq 0$, let us first consider $j=0$. Paralleling the proof of Theorem 3.2, we see that

$$
\pi_{0}=\lim _{\lambda \rightarrow 0^{+}} \lambda \tilde{r}_{00}(\lambda)=(c a-b)\left[c a-b+\mu_{1}+\sum_{k=1}^{c-1} r_{k}(c-k) a\right]^{-1},
$$

whilst for $j \geq 1$, from (3.3), it follows that

$$
\pi_{j}=\lim _{\lambda \rightarrow 0^{+}} \lambda \tilde{r}_{0 j}(\lambda)=\pi_{0} \sum_{i=1}^{\infty} h_{i} \int_{0}^{\infty} p_{i j}^{*}(t) \mathrm{d} t=\pi_{0} r_{j}
$$


whence, by (2.11) and letting $\lambda \rightarrow 0^{+}$in every equation of (2.7), we immediately obtain (3.6). Thus, on applying (2.9) and (2.10), we have

$$
\begin{aligned}
\Pi(s) & =\pi_{0}\left[1+\lim _{\lambda \rightarrow 0^{+}} \sum_{i=1}^{\infty} h_{i} \sum_{j=1}^{\infty} \phi_{i j}^{*}(\lambda) s^{j}\right] \\
& =\pi_{0}\left[1+\frac{s(h-H(s))}{U(s)}\right]+\frac{1}{U(s)} \sum_{k=1}^{c-1} \pi_{k} s^{k}(c-k) a(1-s) .
\end{aligned}
$$

The proof is complete.

Remark 3.1. Known queueing probability generating functions can be easily extracted from this general conclusion as special cases. For example, if $h_{1}=b, h_{k}=0, k \geq 2$, and let $\rho=b / a$, then we recover the ordinary $\mathrm{M} / \mathrm{M} / c$ queue; since $h=b, \mu_{1}=b$, and $H(s)=b s$, (3.5) reduces to

$$
\Pi(s)=\frac{a}{c a-b s} \sum_{k=0}^{c-1} \pi_{k} s^{k}(c-k)
$$

where

$$
\pi_{0}=\left[\sum_{k=0}^{c} \frac{\rho^{k}}{k !}+\frac{\rho^{c+1}}{c !(c-\rho)}\right]^{-1}, \quad \pi_{k}= \begin{cases}\frac{\rho^{k}}{k !} \pi_{0}, & k=1,2, \ldots, c-1, \\ \frac{\rho^{k}}{c^{k-c} c !} \pi_{0}, & k \geq c .\end{cases}
$$

If $c=1$, then we obtain the solution determined by Chen and Renshaw [6].

From (3.5), we obtain the following corollary which illustrates other important queueing features.

Corollary 3.1. The equilibrium queue size, $N$, has expectation

$$
\mathbb{E}(N)=\pi_{0}\left[\frac{b \mu_{1}}{(b-c a)^{2}}-\frac{2 \mu_{1}+H^{\prime \prime}(1)}{2(b-c a)}\right]+\sum_{k=1}^{c-1} \pi_{k}(c-k) a\left[\frac{b}{(b-c a)^{2}}-\frac{k}{b-c a}\right]
$$

if and only if $H^{\prime \prime}(1)$ is finite. The equilibrium waiting queue size, $L_{\mathrm{w}}$, has expectation

$$
\mathbb{E}\left(L_{\mathrm{w}}\right)=\pi_{0}\left[\frac{b \mu_{1}}{(b-c a)^{2}}-\frac{2 \mu_{1}+H^{\prime \prime}(1)}{2(b-c a)}+c\right]+\sum_{k=1}^{c-1} \pi_{k}(c-k)\left[a\left(\frac{b}{(b-c a)^{2}}-\frac{k}{b-c a}\right)+1\right]-c
$$

if and only if $H^{\prime \prime}(1)$ is finite. Here, $\pi_{k}, 0 \leq k \leq c-1$, is given in Theorem 3.3.

\section{The $M / M / c$ queue with resurrection and mass exodus}

In this section we consider the general case that $\beta>0$, the $q$-matrix $Q$ now takes the form of (1.1), i.e. $Q=Q^{*}+Q_{s}+Q_{d}$, where $Q_{s}$ and $Q_{d}$ are defined by (1.3) and (1.4). Let $R(\lambda)=\left\{r_{i j}(\lambda) ; i, j \geq 0\right\}$ denote the $Q$-resolvent. Note that the properties of this process are substantially different from those developed for the $\beta=0$ case in Section 3 . Using the resolvent decomposition theorem (see Chen and Renshaw [5]), we obtain the following theorem. 
Theorem 4.1. For $R(\lambda)=\left\{r_{i j}(\lambda) ; i, j \geq 0\right\}$, we have

$$
\begin{aligned}
& r_{00}(\lambda)=\left[\lambda+\lambda \sum_{i=1}^{\infty} \sum_{j=1}^{\infty} h_{i} \phi_{i j}^{*}(\lambda+\beta)\right]^{-1}, \\
& r_{i 0}(\lambda)=r_{00}(\lambda)\left[a \phi_{i 1}^{*}(\lambda+\beta)+\beta \sum_{k=1}^{\infty} \phi_{i k}^{*}(\lambda+\beta)\right], \quad i \geq 1, \\
& r_{0 j}(\lambda)=r_{00}(\lambda) \sum_{i=1}^{\infty} h_{i} \phi_{i j}^{*}(\lambda+\beta), \quad j \geq 1, \\
& r_{i j}(\lambda)=\phi_{i j}^{*}(\lambda+\beta)+r_{i 0}(\lambda) \sum_{k=1}^{\infty} h_{k} \phi_{k j}^{*}(\lambda+\beta), \quad i, j \geq 1,
\end{aligned}
$$

where $\Phi^{*}(\lambda)=\left\{\phi_{i j}^{*}(\lambda) ; i, j \geq 0\right\}$ is the $Q^{*}$-resolvent given in Theorem 2.2.

On paralleling (2.10) with $\beta>0$, we have

$$
\sum_{k=1}^{\infty} \phi_{i k}^{*}(\lambda+\beta)=\frac{1-u(\lambda+\beta)^{i}+\sum_{k=1}^{c-1} \phi_{i k}^{*}(\lambda+\beta) u(\lambda+\beta)^{k-1}(c-k) a(1-u(\lambda+\beta))}{\lambda+\beta} .
$$

Since

$$
a \phi_{i 1}^{*}(\lambda+\beta)=(\lambda+\beta) \phi_{i 0}^{*}(\lambda+\beta)=u(\lambda+\beta)^{i}-M_{i}(\lambda),
$$

where

$$
M_{i}(\lambda)=\sum_{k=1}^{c-1} \phi_{i k}^{*}(\lambda+\beta) u(\lambda+\beta)^{k-1}(c-k) a(1-u(\lambda+\beta)),
$$

then it follows that (4.2) has the much simpler form

$$
r_{i 0}(\lambda)=r_{00}(\lambda) \frac{\lambda u(\lambda+\beta)^{i}-\lambda M_{i}(\lambda)+\beta}{\lambda+\beta} .
$$

Moreover, (4.1) reduces to a simple expression involving $H(s)$; namely,

$$
\begin{aligned}
r_{00}(\lambda)=\left[\lambda+\frac{\lambda}{\lambda+\beta}(\right. & H(1)-H(u(\lambda+\beta)) \\
& \left.\left.\quad+\sum_{k=1}^{c-1} u(\lambda+\beta)^{k-1}(c-k) a(1-u(\lambda+\beta)) \sum_{i=1}^{\infty} h_{i} \phi_{i k}^{*}(\lambda+\beta)\right)\right]^{-1} .
\end{aligned}
$$

Paralleling Section 2, define the extinction time $\tau_{0}=\inf \left\{t>0 ; X_{t}=0\right\}$ with $\tau_{0}=\infty$ if $X_{t}>0$ for all $t>0$, and recall that

$$
e_{k}=\mathbb{P}_{r}\left(\tau_{0}<\infty \mid X_{0}=k\right), \quad w_{k}(t)=\mathbb{P}_{r}\left(\tau_{0} \leq t \mid X_{0}=k\right)
$$

denote the extinction probability and distribution function of $\tau_{0}$, starting from $X_{0}=k$, respectively. 
Theorem 4.2. If $h=0$ and $\beta>0$. Then, for any $k \geq 1$,

$$
\begin{aligned}
\int_{0}^{\infty} & \mathrm{e}^{-\lambda t} \mathbb{P}_{r}\left(\tau_{0} \leq t \mid X_{0}=k\right) \mathrm{d} t \\
& =\frac{1}{\lambda+\beta}\left(\frac{\beta}{\lambda}+u(\lambda+\beta)^{k}-\sum_{i=1}^{c-1} \phi_{k i}^{*}(\lambda+\beta) u(\lambda+\beta)^{i-1}(c-i) a(1-u(\lambda+\beta))\right),
\end{aligned}
$$

where $u(\lambda)$ is the unique root of $U_{\lambda}(s)=0$ on $[0,1]$ and $\phi_{k i}^{*}(\lambda), k \geq 1,1 \leq i \leq c-1$, is obtained by Theorem 2.1. Moreover, $e_{k}=1, k=1,2, \ldots$, and the mean extinction time is finite and given by

$$
\mathbb{E}\left(\tau_{0} \mid X_{0}=k\right)=\frac{1}{\beta}\left(1-u(\beta)^{k}+\sum_{i=1}^{c-1} \phi_{k i}^{*}(\beta) u(\beta)^{i-1}(c-i) a(1-u(\beta))\right) .
$$

Proof. The Laplace transform (4.6) is precisely (4.4), since for our current case $r_{00}(\lambda)=$ $1 / \lambda$. Hence,

$$
e_{k}=\lim _{\lambda \rightarrow 0^{+}} \frac{\beta+\lambda u(\lambda+\beta)^{k}-\lambda \sum_{i=1}^{c-1} \phi_{k i}^{*}(\lambda+\beta) u(\lambda+\beta)^{i-1}(c-i) a(1-u(\lambda+\beta))}{\lambda+\beta}=1 .
$$

By using (4.6) and the Tauberian theorem, we obtain (4.7). The proof is complete.

We now consider the $h>0$ case. First, by Theorem 2.1, we give the following important lemma.

Lemma 4.1. For $\left(p_{i j}^{*}(t) ; i, j \geq 0\right)$ and $\left(\phi_{i j}^{*}(\lambda) ; i, j \geq 0\right)$ given in Section 2 , denote $L_{j}(\lambda)=$ $\sum_{i=1}^{\infty} h_{i} \phi_{i j}^{*}(\lambda), j \geq 0$. Then $L_{j}(\lambda), 0 \leq j \leq c-1$, is the unique solution of the following linear equations

$$
\begin{gathered}
-\lambda L_{0}(\lambda)-\sum_{k=1}^{c-1} u(\lambda)^{k-1}\left[B_{c}(u(\lambda))-B_{k}(u(\lambda))\right] L_{k}(\lambda)=-H(u(\lambda)), \\
-\lambda L_{0}(\lambda)+a L_{1}(\lambda)=0 \\
(-b-a-\lambda) L_{1}(\lambda)+2 a L_{2}(\lambda)=-h_{1}, \\
\vdots \\
L_{j-1}(\lambda) b+(-b-j a-\lambda) L_{j}(\lambda)+(j+1) a L_{j+1}(\lambda)=-h_{j}, \\
\vdots \\
L_{c-3}(\lambda) b+[-b-(c-2) a-\lambda] L_{c-2}(\lambda)+(c-1) a L_{c-1}(\lambda)=-h_{c-2},
\end{gathered}
$$

where $u(\lambda), \lambda>0$, is the unique root of $U_{\lambda}(s)=0$ on $[0,1]$. Moreover, all the $L_{j}(\lambda), j \geq 0$, can be obtained.

Theorem 4.3. If $h>0$, then the $Q$-process is always positive recurrent.

Proof. On using (4.5) it is easy to see that $\lim _{\lambda \rightarrow 0^{+}} r_{00}(\lambda)=\infty$, and so the $Q$-process is recurrent. Positive recurrence then follows by taking the limit of (4.5) to form

$$
\lim _{t \rightarrow \infty} p_{00}(t)=\beta\left[\beta+H(1)-H(u(\beta))+\sum_{k=1}^{c-1} L_{k}(\beta) u(\beta)^{k-1}(c-k) a(1-u(\beta))\right]^{-1}>0
$$

The proof is complete. 
The following theorem gives the equilibrium distribution $\left\{\pi_{j} ; j \geq 0\right\}$ of the $Q$-process.

Theorem 4.4. The equilibrium distribution of the $Q$-process is given by

$$
\Pi(s)=\pi_{0}\left[1+\frac{s(H(u(\beta))-H(s))}{U_{\beta}(s)}\right]+\frac{\sum_{k=1}^{c-1} \pi_{k}(c-k) a\left[s^{k}(1-s)-s u(\beta)^{k-1}(1-u(\beta))\right]}{U_{\beta}(s)},
$$

where $U_{\beta}(s)$ is defined in (2.3) and

$$
\pi_{0}=\beta\left[\beta+H(1)-H(u(\beta))+\sum_{k=1}^{c-1} L_{j}(\beta) u(\beta)^{k-1}(c-k) a(1-u(\beta))\right]^{-1},
$$

$\pi_{j}=\pi_{0} L_{j}(\beta), j \geq 1$, and $L_{j}(\beta), j \geq 1$, can be obtained by Lemma 4.1.

Proof. First note that (4.10) is precisely (4.8), whilst, for $j \geq 1$,

$$
\pi_{j}=\lim _{\lambda \rightarrow 0^{+}} \lambda r_{0 j}(\lambda)=\pi_{0} L_{j}(\beta)
$$

since $\phi_{i j}^{*}(\lambda)$ is a continuous function of $\lambda>0$. Whence, from (2.9), (2.10), and (4.11), we obtain (4.9), which completes the proof.

Having obtained the equilibrium distribution, we immediately derive the following corollary regarding the queue size.

Corollary 4.1. The equilibrium queue size, $N$, has expectation

$$
\begin{aligned}
\mathbb{E}(N)= & \pi_{0} \frac{\left(H(u(\beta))-h-\mu_{1}\right)(-\beta)-(H(u(\beta))-h)(b-c a-\beta)}{\beta^{2}} \\
& +\frac{\sum_{k=1}^{c-1} \pi_{k}(c-k) a\left[\beta+u(\beta)^{k-1}(1-u(\beta))(b-c a)\right]}{\beta^{2}}
\end{aligned}
$$

and the equilibrium waiting queue size, $L_{\mathrm{w}}$, has expectation

$$
\begin{aligned}
\mathbb{E}\left(L_{\mathrm{w}}\right)= & \pi_{0}\left[\frac{\left(H(u(\beta))-h-\mu_{1}\right)(-\beta)-(H(u(\beta))-h)(b-c a-\beta)}{\beta^{2}}+c\right] \\
& +\frac{\sum_{k=1}^{c-1} \pi_{k}(c-k)\left[a\left(\beta+u(\beta)^{k-1}(1-u(\beta))(b-c a)\right)+\beta^{2}\right]}{\beta^{2}}-c,
\end{aligned}
$$

where $\pi_{k}, 0 \leq k \leq c-1$, is given in Theorem 4.4.

\section{Acknowledgements}

The authors would like to thank the anonymous referee and the editor for their valuable suggestions, which have extremely improved this paper. This work was substantially supported by the National Natural Sciences Foundations of China (grant no. 11371374), Research Fund for the Doctoral Program of Higher Education of China (grant no. 20110162110060), and a Research Fund of CSU (grant no. 2010QYZD001). 


\section{References}

[1] Anderson, W. J. (1991). Continuous-Time Markov Chains: An Applications-Oriented Approach. Springer, New York.

[2] Artalejo, J. R. (2000). G-networks: a versatile approach for work removal in queueing networks. Europ. J. Operat. Res. 126, 233-249.

[3] Asmussen, S. (2003). Applied Probability and Queues, 2nd edn. Springer, New York.

[4] BAYER, N. AND Boxma, O. J. (1996). Wiener-Hopf analysis of an $M / G / 1$ queue with negative customers and of a related class of random walks. Queueing Systems Theory Appl. 23, 301-316.

[5] Chen, A. Y. And Renshaw, E. (1990). Markov branching processes with instantaneous immigration. Prob. Theory Relat. Fields 87, 209-240.

[6] Chen, A. And Renshaw, E. (1997). The $M / M / 1$ queue with mass exodus and mass arrivals when empty. J. Appl. Prob. 34, 192-207.

[7] Chen, A. AND Renshaw, E. (2004). Markovian bulk-arriving queues with state-dependent control at idle time. Adv. Appl. Prob. 36, 499-524.

[8] Chen, A., Pollett, P., Li, J. AND Zhang, H. (2010). Markovian bulk-arrival and bulk-service queues with state-dependent control. Queueing Systems 64, 267-304.

[9] Chen, M. F. (1992). From Markov Chains to Nonequilibrium Particle Systems. World Scientific, River Edge, NJ.

[10] Chen, M.-F. (2005). Eigenvalues, Inequalities, and Ergodic Theory. Springer, London.

[11] Di Crescenzo, A., Giorno, V., Nobile, A. G. And Ricciardi, L. M. (2008). A note on birth-death processes with catastrophes. Statist. Prob. Lett. 78, 2248-2257.

[12] Dudin, A. N. AND Karolik, A. V. (2001). BMAP/SM/1 queue with Markovian input of disasters and noninstantaneous recovery. Performance Evaluation 45, 19-32.

[13] Gelenbe, E. (1991). Product-form queueing networks with negative and positive customers. J. Appl. Prob. 28, $656-663$.

[14] Gelenbe, E., Glynn, P. and Sigman, K. (1991). Queues with negative arrivals. J. Appl. Prob. 28, $245-250$.

[15] Gross, D. AND Harris, C. M. (1985). Fundamentals of Queueing Theory, 2nd edn. John Wiley, New York.

[16] JaIN, G. AND Sigman, K. (1996). A Pollaczek-Khintchine formula for $M / G / 1$ queues with disasters. J. Appl. Prob. 33, 1191-1200.

[17] LI, J. AND ChEN, A. (2006). Markov branching processes with immigration and resurrection. Markov Process. Relat. Fields 12, 139-168.

[18] Parthasarathy, P. R. and Krishna Kumar, B. (1991). Density-dependent birth and death process with state-dependent immigration. Math. Comput. Modelling 15, 11-16.

[19] Zeifman, A. AND Korotysheva, A. (2012). Perturbation bounds for $M_{t} / M_{t} / N$ queue with catastrophes. Stoch. Models 28, 49-62. 